\title{
Solitary Fibrous Tumor of the Larynx
}

\author{
Lester D. R. Thompson · Yevgeniy Karamurzin • \\ Mark Li-cheng Wu $\cdot$ Jason H. Kim
}

Received: 22 January 2008/Accepted: 19 February 2008/Published online: 15 March 2008

(c) Humana 2008

\begin{abstract}
Background True mesenchymal, non-cartilaginous neoplasms of the larynx are rare. Extrapleural solitary fibrous tumor (SFT) is a localized neoplasm characterized by proliferation of thin-walled vessels and collagen-producing cells and is considered within the "hemangiopericytoma-solitary fibrous tumor" spectrum. SFT primary in the larynx is exceptional. Design Case report set in a comparison with other cases reported in the English literature (MEDLINE 1966 to 2007). Results A 49year old white male presented with difficulty breathing, progressive over the past 2 years. He denied dysphagia and weight loss. Past medical history was significant for asthma. He denied cigarette smoking or alcohol abuse. There were no cervical deformities on physical exam. Fiberoptic laryngoscopy was performed upon stabilization of respiratory function. A smooth, round, submucosal mass measuring $2.3 \mathrm{~cm}$ in greatest diameter arising from the inferior surface of left true vocal cord was causing near total obstruction of the endolaryngeal space. The mass was
\end{abstract}

L. D. R. Thompson ( $\square)$

Woodland Hills Medical Center, Department of Pathology, Southern California Permanente Medical Group, 5601 De Soto Avenue, Woodland Hills, CA 91365, USA

e-mail: Lester.D.Thompson@kp.org

Y. Karamurzin · M. L. Wu

Irvine School of Medicine, Department of Pathology, University of California, Irvine, CA, USA

e-mail: ykaramur@uci.edu

M. L. Wu

e-mail: mlwu@uci.edu

J. H. Kim

Irvine School of Medicine, Department of Head and Neck

Surgery, University of California, Irvine, CA, USA

e-mail: kimjason@uci.edu excised. The surface mucosa was intact and unremarkable. A cellular, spindle cell neoplasm was arranged in loose fascicles, associated with heavy collagen fiber deposition. The collagen was wiry and heavy. Cells were bland with cytoplasmic extensions. The nuclei were vesicular to hyperchromatic and elongated with inconspicuous nucleoli. Vessels were prominent and delicate, with patulous spaces. Mitotic figures were easily identified, but atypical forms were not present. The cells were strongly and diffusely immunoreactive with $\mathrm{CD} 34$ and bcl-2, while non-reactive with cytokeratin, EMA, actin, ALK-1, S100, desmin, and CD117. These findings confirmed a diagnosis of extraplural solitary fibrous tumor. Without further disease, the patient is alive without evidence of disease, 12 months after surgery. Conclusions The characteristic histologic pattern of solitary fibrous tumor can be noted in extrapulmonary locations. Development in the larynx is uncommon, but the tumor presents as a polypoid mass with characteristic histologic and immunophenotypic features. Conservative local excision is the treatment of choice to yield an excellent prognosis.

Keywords Solitary fibrous tumor $\cdot$ Larynx $\cdot$ Benign neoplasm - Mesenchymal tumor - Hemangiopericytoma . Spindle cell squamous cell carcinoma - True vocal cord . Immunohistochemistry $\cdot$ CD34 - bcl-2 · Fibroma ·

Prognosis $\cdot$ Surgery

\section{Introduction}

Solitary fibrous tumor (SFT) is a localized mesenchymal neoplasm characterized by proliferation of thin-walled vessels and collagen-producing cells. This entity is considered a spectrum of hemangiopericytoma-solitary 
fibrous tumor, a tumor category group fraught with diagnostic mystery [1]. The neoplasm was first described in 1931 by Dr. Klemperer within the pleura [2], but has come to be recognized in a variety of locations; hence the use of the term "extrapleural" or "extrathoracic" solitary fibrous tumor [3, 4]. SFT manifests along a benign to malignant spectrum, but in the vast majority of cases characterized by slow growth clinically and a benign histology [4, 5]. While the tumor commonly develops within a serosal space [6], the tumor has no relationship to mesothelium, even though it has been called "submesothelial fibroma" and "solitary fibrous mesothelioma." [7, 8] Diagnosis of SFT is based on architectural, cytomorphologic, and immunohistochemical findings. Tumors have been described in nearly every organ of the body (orbit, meninges, liver, salivary gland, mediastinum, external auditory canal, thyroid, tongue, infratemporal fossa [1, 3, 4, 9-11]), but laryngeal SFT is exceptionally rare, with only seven case reports identified (Table 1) [8, 12-17]. Mesenchymal tumors of the larynx are rare, with SFT even more uncommon. The results of the cases reported in the literature were analyzed in comparison to the present case.

\section{Materials and Methods}

The patient records were reviewed, including the history and physical, radiographic studies, laboratory values, histology, immunohistochemistry studies, and follow-up management. The data was compared to a compilation of cases obtained by a review of larynx case publications in English (MEDLINE 1966 to 2007). Cases specifically reported as "hemangiopericytoma, solitary fibrous tumor" were included in the review (Table 1).

Immunophenotypic analysis was performed on suitable material by a standardized Envision ${ }^{\mathrm{TM}}$ method employing $4 \mu \mathrm{m}$-thick, formalin fixed, paraffin embedded sections. A pertinent, commercially available immunohistochemical antibody panel used including bcl-2, CD34 (anti-HPCA-1), keratin (AE1/AE3), EMA, CK7, CD117, smooth muscle actin, muscle specific actin, desmin, CD99, MIB-1 (Ki-67), S-100 protein, HMB-45, ALK-1, and vimentin. The analysis was performed on a single block. Proteolytic antigen retrieval was performed, as required, by predigestion for 3 min with $0.05 \%$ Protease VIII (Sigma Chemical Co., St. Louis, MO) in a $0.1 \mathrm{M}$ phosphate buffer, $\mathrm{pH}$ of 7.8 , at $37^{\circ} \mathrm{C}$. Heat induced epitope retrieval was performed, as required, by using formalin-fixed, paraffin-embedded tissue treated with a buffered citric acid solution pH 6.0 (Citra, Dako Corporation, Carpinteria, CA) and heated for $20 \mathrm{~min}$ in a steamer. Following this, the sections were allowed to cool at room temperature in a citric acid buffer solution for 45 min before continuing the procedure. Standard positive controls were used throughout, with serum used as the negative control.

\section{Case Report}

\section{Clinical Presentation}

A 49-year-old white male presented with difficulty breathing progressively getting worse for the last 2 years. Initial patient evaluation was triggered by acute respiratory distress due to airway obstruction which required emergent tracheostomy. He denied dysphagia and weight loss. Past medical history was significant for asthma since childhood, gunshot wound to the abdomen 23 years ago with subsequent tracheostomy, and remote cigarette smoking (discontinued 15 years ago). No cervical masses or deformities were seen on physical examination. Fiberoptic laryngoscopy was performed upon stabilization of respiratory function. It revealed a smooth, round, submucosal mass measuring $2.3 \mathrm{~cm}$ in greatest diameter, arising form inferior surface of left true vocal cord and causing near total obstruction of the lumen. The remaining laryngeal structures were unremarkable.

Computed tomography scans demonstrated an enhancing polypoid mass occluding the endolaryngeal space (Fig. 1). The mass was attached by a pedicle to the inferior portion of the left true vocal cord. The surface was smooth and there was no destruction of the laryngeal tissues, although significant occlusion was noted (Fig. 1, right).

A biopsy was performed, with an initial diagnosis of low grade malignant hemangiopericytoma. Shortly thereafter, the patient underwent an excision. A mucosal flap was elevated, revealing the submucosal topography of the mass. The tumor bled significantly during excision. The "polypectomy" showed a free stalk without destructive growth into the laryngeal structures.

Pathologic Features

\section{Macroscopic}

The initial specimen consisted of multiple tan-brown fragments of tissue with rubbery consistency ranging from 0.1 to $1.7 \mathrm{~cm}$ in greatest diameter. The second specimen was a single polypoid, tan-brown, solid, firm to rubbery mass measuring $1.3 \times 1.1 \times 0.7 \mathrm{~cm}$.

\section{Microscopic}

Histomorphologic evaluation reveals a polypoid mass subtending an intact epithelium (Fig. 2). The mesenchymal neoplasm is composed of a variably cellular proliferation of bland spindle-shaped cells lacking any pattern of growth 


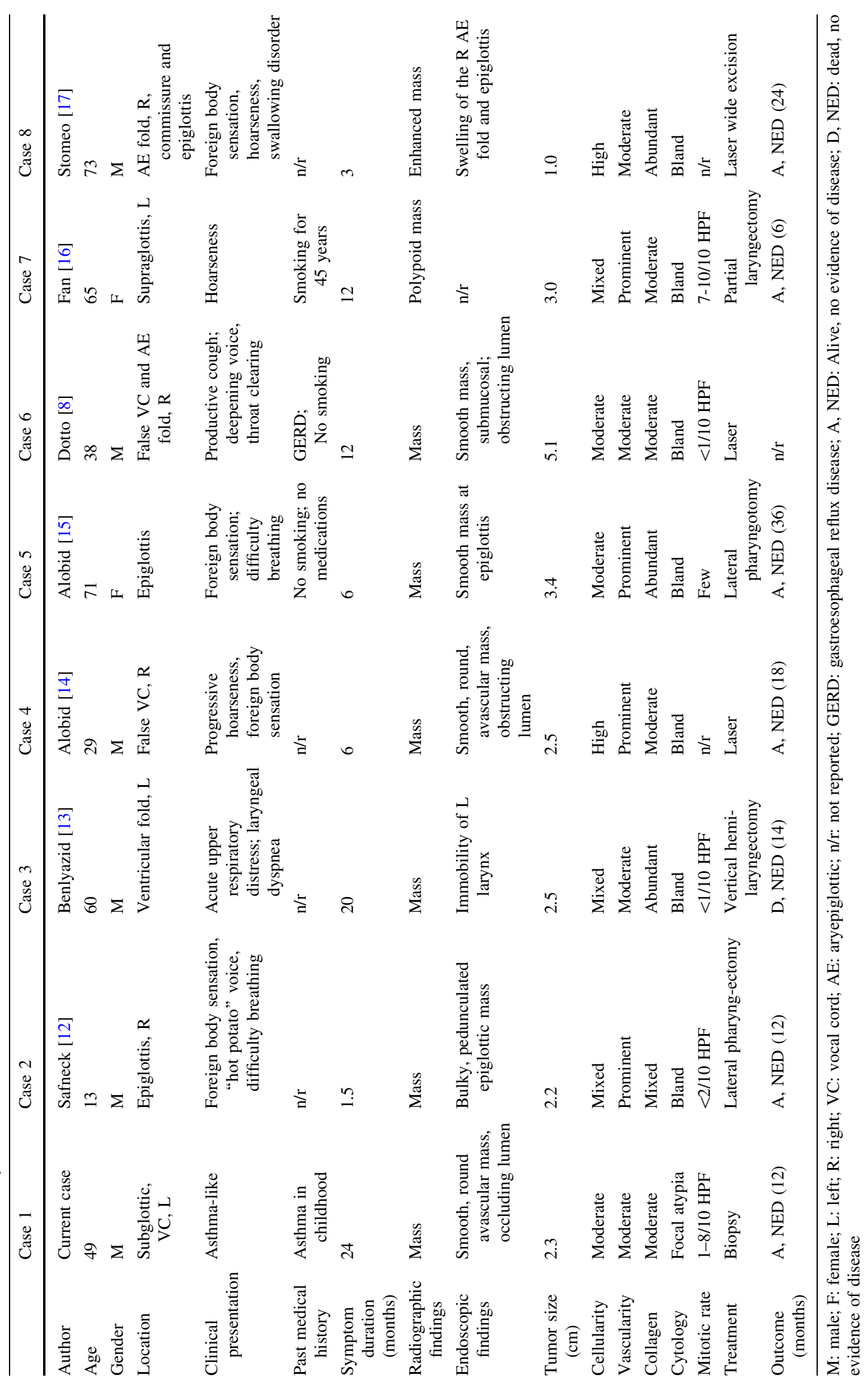



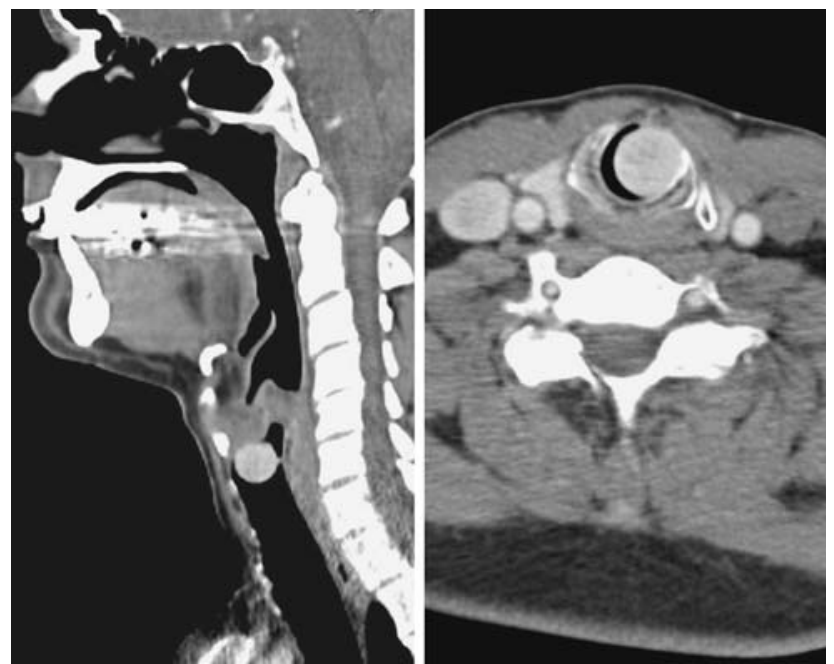

Fig. 1 Post-contrast computed tomography scans. Left: a saggittal view demonstrates a polypoid mass occluding the lumen of the larynx. Right: the cross section shows a slightly enhancing soft tissue density mass nearly completely occluding the larynx. There is no invasion or destruction of the laryngeal structure

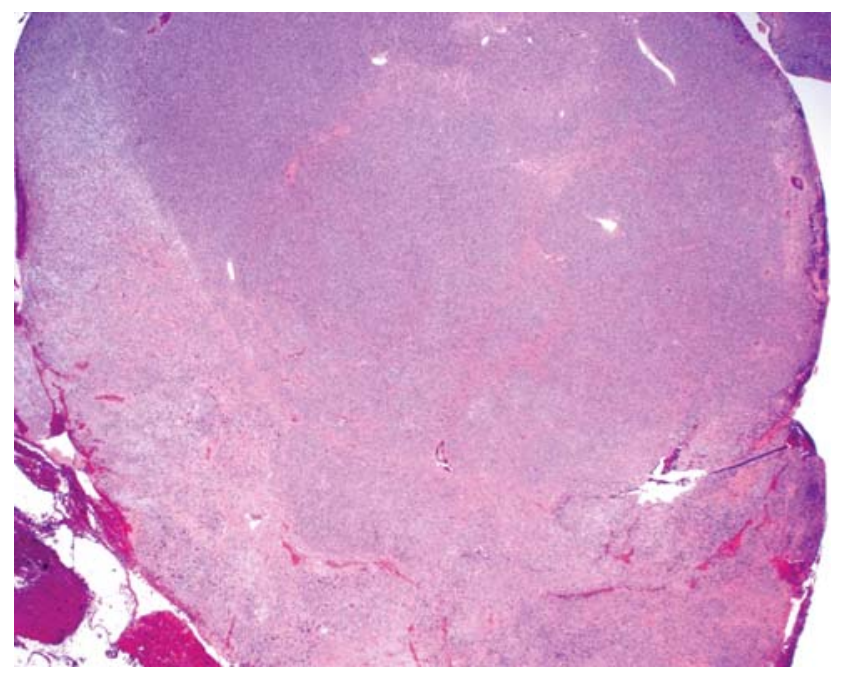

Fig. 2 The polypoid mass shows a variably cellular spindle cell proliferation in a background of collagenized stroma

and associated with "ropey" keloidal collagen bundles and interlaced thin-walled vascular spaces (Figs. 3 and 4). The vascular component is represented by thin-walled dilated capillaries to large patulous spaces. A "hemangiopericytoma-like" vascularity ("staghorn pattern") was noted, presumably responsible for initial misclassification of the tumor (Fig. 3).

The cellularity ranged from hypocellular regions with prominent dense collagenous background to areas with high cellularity (Fig. 4). Cellular pleomorphism was minimal, with only focally enlarged nuclei. The cells showed scant, indistinct faintly eosinophilic cytoplasm, and either

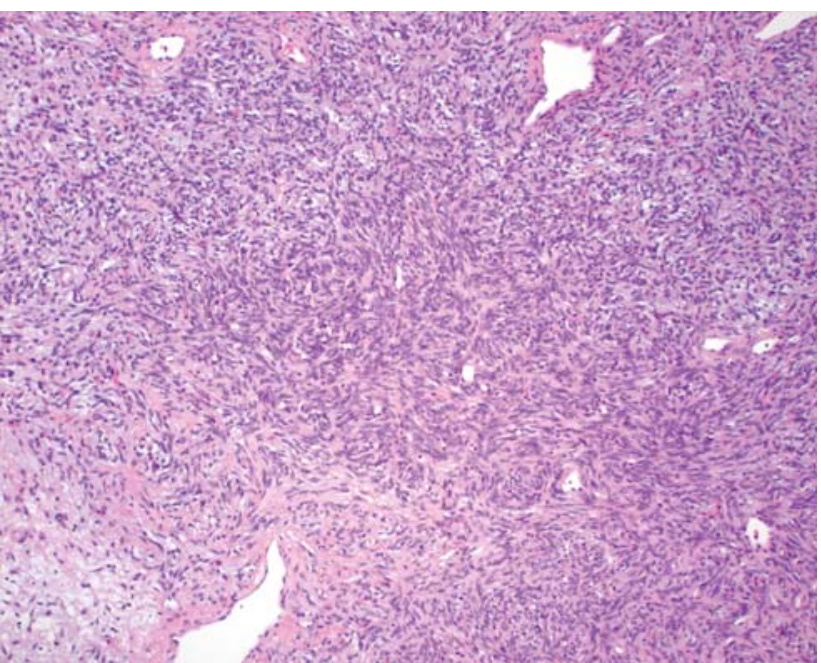

Fig. 3 Short fascicles of cytologically bland spindled cells set in a fibrous stroma. Note the variably sized vessels, one of which is patulous (bottom)

elongate dense nuclei or vesicular nuclei with inconspicuous nucleoli. Mitotic figures are noted, focally increased (8/ 10 high power fields; Fig. 4, right lower), but atypical mitotic forms were not present.

\section{Immunohistochemical Results}

By immunohistochemical analysis, the neoplastic cells were strongly and diffusely immunoreactive with CD34, bcl-2 (Fig. 5) and vimentin; isolated cells were positive for CD99 and Ki-67, the latter supporting a low proliferation index (Fig. 6). The lesional cells were non-reactive with keratin, EMA, CK7, smooth muscle actin, muscle specific actin, desmin, CD117, ALK-1, S100, and HMB-45.

\section{Clinical Treatment and Patient Outcome}

After the initial biopsy and further polypectomy (excision), the patient was re-evaluated and shown to have no residual tumor. No further treatment was performed. He has been followed prospectively for the past 12 months, without any symptoms and no recurrent disease.

\section{Discussion}

Solitary fibrous tumors (SFTs) show considerable histologic variability which contributes to the difficulty of diagnosing this neoplasm in extrathoracic sites, such as the larynx. The exceptional rarity of this tumor is highlighted by the limited number of reported cases in the English literature (Table 1). In combination with our case, we can summarize the clinical, radiologic, endoscopic, 
Fig. 4 This composite illustrations shows the variation in cellularity and architectural patterns. Collagen deposition and spindled cells are noted in all patterns. Mitotic figures are shown, with four in a single high power field (although this was a focal finding)
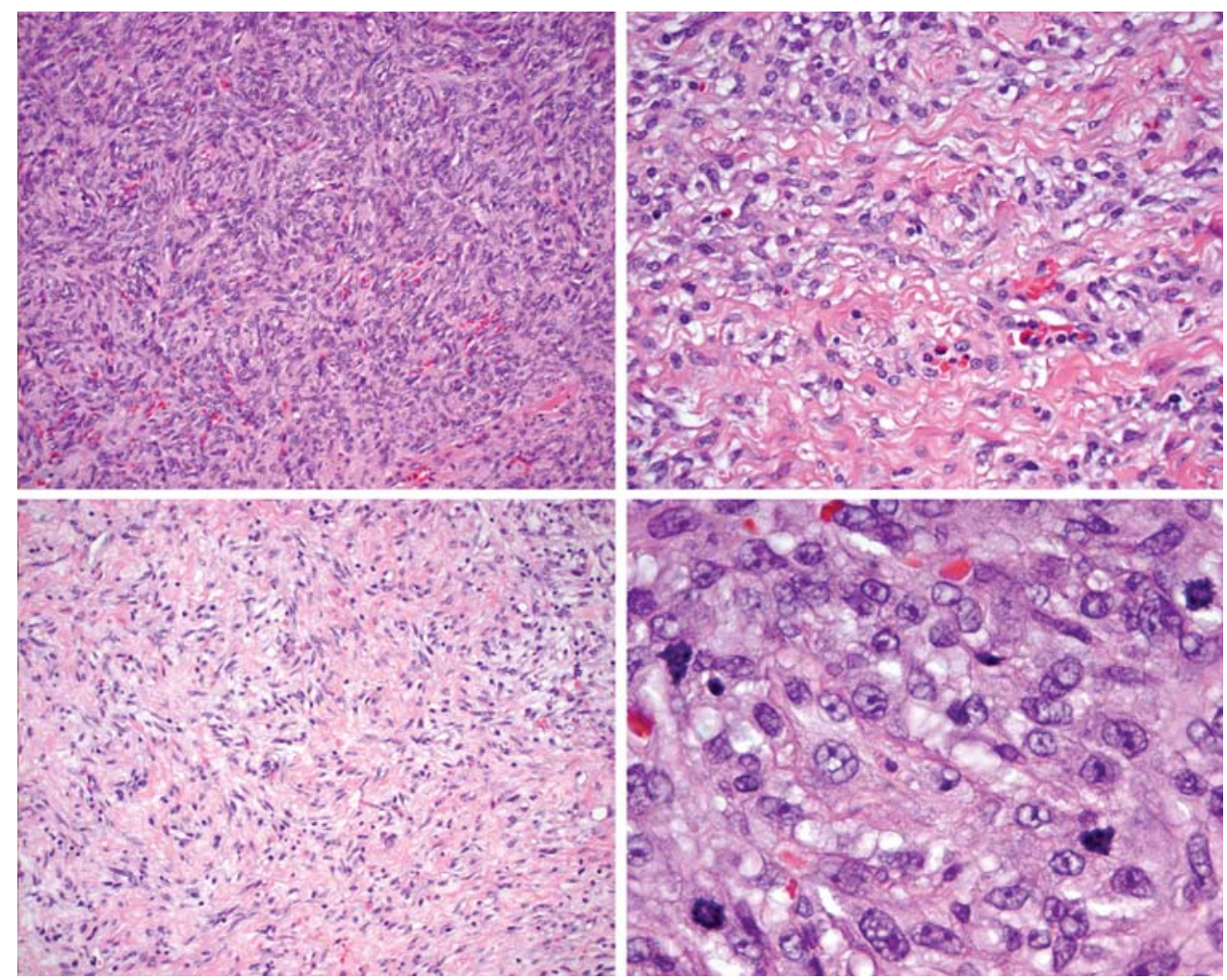
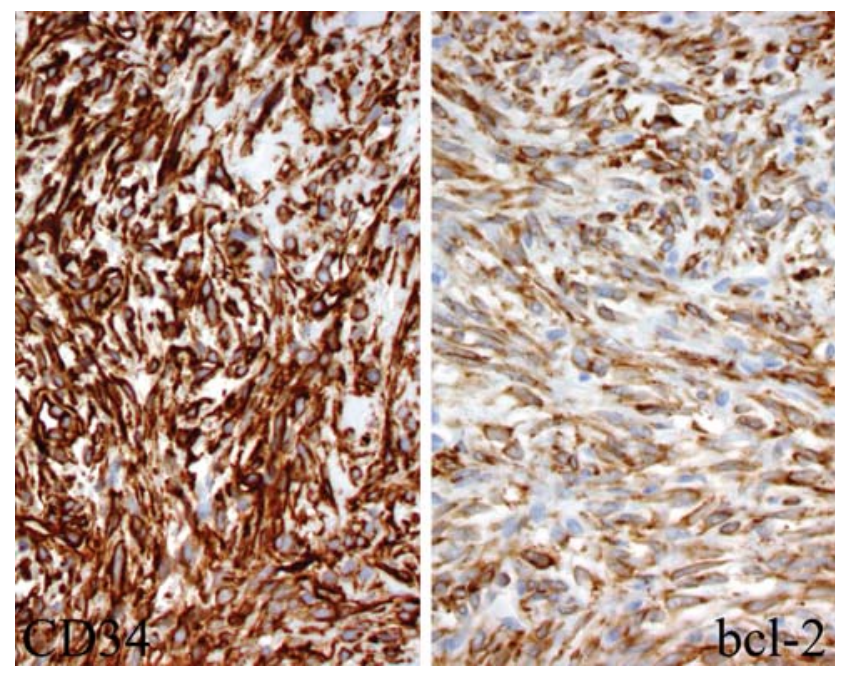

Fig. 5 The lesional cells are strongly immunoreactive with both CD34 (left) and bcl-2 (right)

macroscopic, microscopic, immunophenotypic, and outcome results of this rare tumor to try to yield some generalizations about larynx SFTs.

About $40 \%$ of all SFTs develop in subcutaneous tissues or other deep soft tissues, particularly in the head and neck (orbit especially), thoracic wall, and abdominal cavity [1, $3,4,7,11]$. Although the neoplasm is extremely rare in the larynx, males are affected more commonly than females (6:1). This finding is different from the equal gender

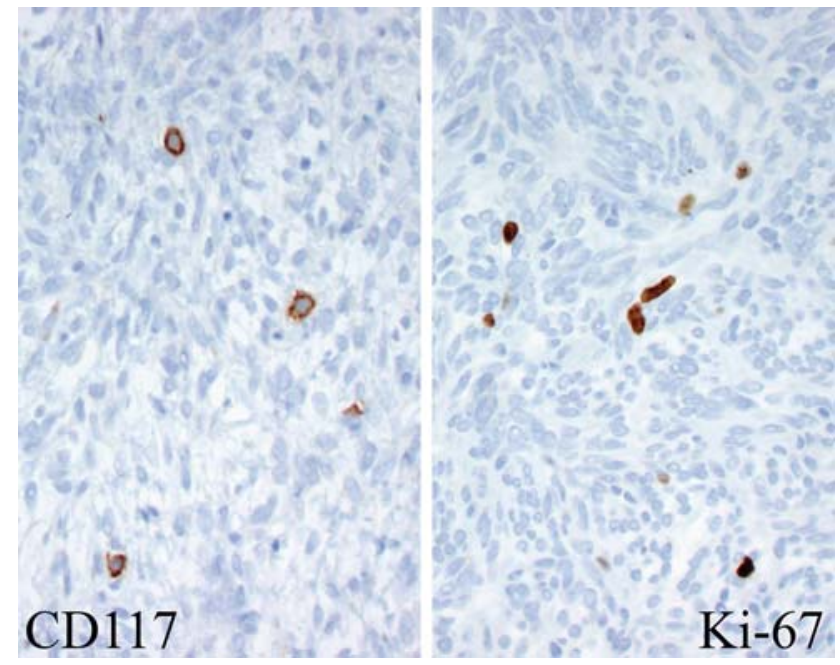

Fig. 6 Left: mast cells are highlighted by CD117, although the tumor cells are negative. Right: Ki-67 highlights the proliferating nuclei, although the overall rate is $<3-4 \%$

distribution in other anatomic sites [1, 4]. All ages are affected, but the average age at presentation is 46.7 years, suggesting the disease is more common in adults, similar to other soft tissue sites $[1,4]$. In fact, if the only child is eliminated [12], the average age becomes 52.3 years, similar to extrathoracic disease in other reports [4]. There is no significant difference in age at presentation for males versus females. 
All of the patients had a similar, non-specific clinical presentation of progressive hoarseness, foreign body sensation, changes in phonation, difficulty breathing, cough, and/or throat clearing. Since the tumor is considered slow growing, symptoms are frequently present for a long time. Interestingly, a number of the patients presented with acute respiratory embarrassment due to airway obstruction, requiring urgent intervention, although the acute event was often the culmination of many months of symptoms (average, 11.2 months) [13, 17]. Extrathoracic SFTs seem to have a greater change of being symptomatic in comparison to their thoracic counterparts [4]. The foreign body sensation was sometimes body position-dependent, suggesting a "ball-valve" effect on the airway [12]. The "hot potato" voice quality is sometimes associated with a supraglottic mass $[8,12]$. Only two of the patients reported a smoking history and one patient also had childhood asthma [16]. No other environmental or inherited etiologic factor was gleaned from the reports, suggesting that more cases would need to be evaluated before a definitive etiologic agent can be determined. No paraneoplastic syndromes have been reported with larynx SFT, although hypoglycemia has been identified in association with tumors of other anatomic sites $[1,4,15]$.

Radiologic studies of greatest value seem to be either post-contrast computed tomography studies or magnetic resonance imaging $[12,13,15,17]$. The usual radiologic findings are of an intensely contrast enhancing, polypoid to spherical mass arising from the submucosal tissue, producing laryngeal obstruction. A pedunculated mass is the most common finding [12]. There is a lack of destructive growth or penetration into the deeper soft tissues of the larynx. The contrast enhancement supports the histologic findings of a richly vascularized neoplasm. Laryngoscopic findings also show a smooth, non-mucosal based, avascular, polypoid or pedunculated mass protruding into and partially to completely occluding the laryngeal lumen. A swelling may also be noted. Unilateral vocal cord immobility is occasionally identified [13].

This case is the only lesion which did not develop in the supraglottic or glottic space, although the pedicle of attachment was to the true vocal cord. The epiglottis, ventricular fold, aryepiglottic fold, vocal fold, or the commissure are the most frequently involved anatomic subsites within the larynx. However, prolapse into the subglottis can be seen [13]. These parts of the larynx are the areas which have the greatest amount of submucosal soft tissue when compared to the true vocal cords (glottis) or subglottic space, perhaps suggesting the cell of origin is within the mesenchymal soft tissue.

Macroscopically, the tumors are polypoid, frequently attached by a stalk or pedicle. The tumors are very well circumscribed or encapsulated. Tumors range from 1 to
$5 \mathrm{~cm}$ (mean, $2.6 \mathrm{~cm}$ ). However, airway compromise results in immediate clinical attention or intervention, suggesting that tumors of the larynx are clinically recognized at a much smaller size than their soft tissue or visceral organ counterparts, which have a median size of between 5 and $8 \mathrm{~cm} \mathrm{[1,4].} \mathrm{The} \mathrm{tumors} \mathrm{are} \mathrm{homogenous,} \mathrm{white-gray} \mathrm{to}$ tan, solid, firm masses.

The pathologic diagnosis of solitary fibrous tumor rests on a combination of architectural, cytomorphologic, and immunophenotypic features, as there are no discriminating ultrastructural or immunohistochemical features $[1,15$, 18]. By definition, it is a mesenchymal tumor of probable fibroblastic type which shows a prominent hemangiopericytoma-like branching vascular pattern [1]. Microscopic evaluation reveals a low-grade mesenchymal neoplasm with a "patternless" distribution of hypo- and hypercellular areas intermingled with dense keloid-like and loose collagen fibers. The tumor may show a variety of growth "patterns," including storiform, herringbone, neural and hemagiopericytic_admixed with sclerosis or collagen. However, no one "pattern" is unique to the tumor. The spindled cells are separated by endothelial-lined, dilated and angulated vascular spaces, focally arranged in a "hemangiopericytoma-like" pattern. The vessels range from capillaries to large patulous vessels, but usually do not have significant muscular walls. There is no cytologic atypia within the endothelial cells. Further, cytologic atypia is generally absent in the histologically bland spindled cells. Focal variability can be seen, but in general nuclear pleomorphism is absent or limited. The cell borders are ill defined, creating a syncytial architecture. The nuclear chromatin is usually vesicular and open with inconspicuous nucleoli. Mitotic activity is often easy to identify, although there is a wide range of activity, with up to 10 mitotic figures per 10 high power fields reported $[4,16]$. However, atypical mitotic figures are not identified in this benign tumor. Although necrosis was not present in this case, necrosis has been observed by others, but usually only focal $[4,16]$. Malignant SFTs show extreme hypercellularity, infiltrative borders, moderate to marked pleomorphism, necrosis and $>4$ mitoses per 10 HPF $[1,3]$. These features were not identified in the present case, although there was focally increased mitotic activity.

Depending upon the degree of cellularity and the particular vascular pattern present, immunohistochemical support for the diagnosis may be required. Uniquely in this anatomic site, SFT reacts with vimentin, CD34, bcl-2, and CD99. The combination of bcl-2 and CD34 has been reported to be consistently identified in SFT, a finding helpful in establishing the diagnosis [18, 19]. CD34 is a cell adhesion transmembrane glycoprotein found on the surface of many cells, showing myofibroblastic differentiation, hematologic progenitor cells, vascular endothelium, 
and dendritic cells. blc- 2 is a molecule involved in the control of programmed cell death. This marker is positive in schwannoma, synovial sarcoma, and gastrointestinal stromal tumor, while not in most sarcomas [19]. A host of other epithelial, mesenchymal, Schwannian, melanocytic and vascular markers are usually non-reactive, findings helpful in making a definitive separation between SFT and lesions within the differential diagnosis [7]. Notably, SFT in other anatomic sites may express EMA, smooth muscle actin and even limited S-100 protein [1, 11], but this has not been identified as yet in larynx SFTs. Ultrastructural studies demonstrate fibroblasts and myofibroblasts which show spindle cells with poorly developed round endoplasmic reticulum, mitochondria and intercellular junctions $[3,11,20]$. Unfortunately, these non-specific findings do not usually help in the surgical pathology diagnostic setting. Likewise, SFTs exhibit a wide range of cytogenetic alterations and chromosomal abnormalities (such as trisomy 8 ) [21, 22]. These alterations tend to be significantly less common in small SFTs [23], perhaps suggesting why they are not identified in laryngeal tumors which are much smaller than their soft tissue or other organ counterparts.

Soft tissue and spindle cell neoplasms of the larynx are rare, but the histologic differential diagnosis covers a wide range of reactive and neoplastic conditions. One of the most important considerations is spindle cell (sarcomatoid) squamous cell carcinoma, but angiosarcoma, hemangiopericytoma, melanoma, fibrous histiocytoma, leiomyoma, schwannoma, inflammatory myofibroblastic tumor, fibromatosis, and other benign spindle cell proliferations are all possible histologic mimics, especially on small biopsies [8, $15,16,18]$. Spindle cell squamous cell carcinoma (SCSCC) tends to develop in male patients in the mid to older decades of life. The tumor is often polypoid and yields a similar clinical presentation as SFT. SCSCC is strongly associated with tobacco and alcohol use. SCSCC tends to have a greater degree of pleomorphism, shows necrosis, has atypical mitotic figures and will often show areas of squamous differentiation (surface or invasive), although sometimes quite focal. Importantly, SCSCC is negative with epithelial markers in approximately $30 \%$ of cases [24]. Therefore, careful review of the architectural patterns, cytologic atypia and additional immunohistochemistry studies may be necessary to make a definitive diagnosis. Angiosarcoma is a vasoformative tumor with freely anastomosing vessels, extravasated erythrocytes, significant cytologic atypia, and endothelial markers beyond just CD34 [25]. Conceptually, hemangiopericytoma and solitary fibrous tumor are now considered to be on a morphologic spectrum, with a greater vascularity in hemangiopericytoma and greater collagen deposition in SFT [1]. It is important to note that a "hemangiopericytoma-like" vascular pattern can be seen in a number of different tumors [26], but the diagnosis of hemangiopericytoma requires specific histologic and immunohistochemical criteria. This distinction may be impossible on a small biopsy and may be surfeit when considered as a single histologic entity. Axiomatic, glomangiopericytoma (sinonasal-type hemangiopericytoma) is a completely different morphologic entity, showing a specific growth pattern, prominent perivascular hyalinization, eosinophils, and a specific smooth-muscle immunophenotype [27]. Primary melanomas of the larynx frequently show a junctional melanocytic proliferation, show remarkable cytologic atypia, may have melanin pigment, and usually show melanocytic differentiation (S100 protein, HMB-45, melan-A) [28]. Primary mesenchymal tumors of the larynx are uncommon, but inflammatory myofibroblastic tumor, leiomyoma, schwannoma (neurilemmoma), neurofibroma, and fibrous histiocytomas are usually histologically and immunophenotypically unique $[29,30]$.

Once the correct diagnosis has been made, conservative surgery is appropriate, although partial laryngectomy and pharyngotomy has been reported [12, 13, 15, 16]. This more aggressive approach may be to maintain functional integrity after removal of the "polypoid" mass. With only limited cases in the literature to review, definitive comments about therapy are difficult to infer. However, it seems that function-preserving, limited excision yields the same outcome as the more radical procedures. Therefore, complete surgical removal yields the best patient outcome. $\mathrm{CO}_{2}$ laser surgery has gained popularity, especially since the rich vascular nature of the neoplasm demands meticulous cauterization and control of bleeding during the surgery $[8,17]$. Radiation and chemotherapy do not play a role in management of head and neck cases [4]. Recurrences have been reported [13], but it may be due to incomplete removal at initial surgery. In fact, an "incisional" biopsy will require follow-up definitive excision. Tumors with positive surgical margins are more likely to recur, although these findings are only reported for extralaryngeal cases [4]. It may be prudent, in a polypoid lesion, to perform a polypectomy at the stalk or point of attachment at initial evaluation to yield the best overall patient management and also give the most material to pathology for histologic and immunophenotypic evaluation. In other anatomic sites, the behavior is unpredictable, with malignant cases having no sequalae with long-term follow-up and histologically benign lesions developing metastatic disease in 6 months $[3,10]$. Tumor size of $>10 \mathrm{~cm}$ has been associated with a more aggressive behavior, but this size is not reached in larynx tumors [4]. With so few cases in the larynx, prudence suggests long term clinical followup is probably warranted as there is no well developed correlation between morphology and behavior. 


\section{Conclusion}

SFT is an exceptional rare tumor in the larynx. This benign neoplasm requires only conservative management with long term clinical follow-up. The unique patternless proliferation of cytological bland cells which may show mitotic figures, set in a collagenized stroma and showing CD34, bcl-2, CD99, and vimentin immunoreactivity is unique in the larynx. Separation from spindle cell (sarcomatoid) squamous cell carcinoma specifically will allow for conservative management, preserving larynx function.

Acknowledgements The opinions or assertions contained herein are the private views of the authors and are not to be construed as official or as reflecting the views of the University of California or Southern California Permanente Medical Group.

\section{References}

1. Guillou L, Fletcher JA, Fletcher CDM, Mandahl N. Extrapleural solitary fibrous tumour and haemangiopericytoma. In: Fletcher CDM, Unni K, Mertens F, editors. Pathology and genetics of tumours of soft tissue and bone, world health organization classification of tumours. Kleihues P, Sobin LH, series editors. Lyon, France: IARC Press, 2002:86-90.

2. Klemperer P, Rabin CB. Primary neoplasms of the pleura. A report of five cases. Arch Pathol 1931;11:385-412.

3. Hasegawa T, Matsuno Y, Shimoda T, et al. Extrathoracic solitary fibrous tumors: their histological variability and potentially aggressive behavior. Hum Pathol 1999;30:1464-73.

4. Gold JS, Antonescu CR, Hajdu C, et al. Clinicopathologic correlates of solitary fibrous tumors. Cancer 2002;94:1057-68.

5. Graadt van Roggen JF, Hogendoorn PCW. Solitary fibrous tumor: the emerging clinicopathologic spectrum of an entity and its differential diagnosis. Curr Diagn Pathol 2004;10:229-35.

6. Scharifker D, Kaneko M. Localized fibrous "mesothelioma" of pleura (submesothelial fibroma): a clinicopathologic study of 18 cases. Cancer 1979;43:627-35.

7. Suster S, Nascimento AG, Miettinen M, et al. Solitary fibrous tumors of soft tissue. A clinicopathologic and immunohistochemical study of 12 cases. Am J Surg Pathol 1995;19:1257-66.

8. Dotto JE, Ahrens W, Lesnik DJ, et al. Solitary fibrous tumor of the larynx: a case report and review of the literature. Arch Pathol Lab Med 2006;130:213-6.

9. Chan JK. Solitary fibrous tumour-everywhere, and a diagnosis in vogue. Histopathology 1997;31:568-76.

10. Brunnemann RB, Ro JY, Ordonez NG, et al. Extrapleural solitary fibrous tumor: a clinicopathologic study of 24 cases. Mod Pathol 1999;12:1034-42.

11. Nielsen GP, O'Connell JX, Dickersin GR, et al. Solitary fibrous tumor of soft tissue: a report of 15 cases, including 5 malignant examples with light microscopic, immunohistochemical, and ultrastructural data. Mod Pathol 1997;10:1028-37.

12. Safneck JR, guacil-Garcia A, Dort JC, et al. Solitary fibrous tumour: report of two new locations in the upper respiratory tract. J Laryngol Otol 1993;107:252-6.

13. Benlyazid A, Lescanne E, Lefrancq T, et al. Solitary fibrous tumour of the larynx: report of a case. J Laryngol Otol 1998;112:286-9.
14. Alobid I, Alos L, Maldonado M, et al. Laryngeal solitary fibrous tumor treated with $\mathrm{CO} 2$ laser excision: case report. Eur Arch Otorhinolaryngol 2005;262:286-8.

15. Alobid I, Bernal-Sprekelsen M, Benitez P, et al. Solitary fibrous tumor of the larynx. Otolaryngol Head Neck Surg 2005;133:1635 .

16. Fan CY, Van Hemert RL, Thomas JR, et al. Atypical solitary fibrous tumor of the larynx. Otolaryngol Head Neck Surg 2006;134:880-2.

17. Stomeo F, Padovani D, Bozzo C, et al. Laryngeal solitary fibrous tumour. Auris Nasus Larynx 2007;34:405-8.

18. Westra WH, Gerald WL, Rosai J. Solitary fibrous tumor. Consistent CD34 immunoreactivity and occurrence in the orbit. Am J Surg Pathol 1994;18:992-8.

19. Chilosi M, Facchettti F, Dei Tos AP, et al. bcl-2 expression in pleural and extrapleural solitary fibrous tumours. J Pathol 1997; 181:362-7.

20. England DM, Hochholzer L, McCarthy MJ. Localized benign and malignant fibrous tumors of the pleura. A clinicopathologic review of 223 cases. Am J Surg Pathol 1989;13:640-58.

21. Krismann M, Adams H, Jaworska M, et al. Patterns of chromosomal imbalances in benign solitary fibrous tumours of the pleura. Virch Arch 2000;437:248-55.

22. Donner LR, Silva MT, Dobin SM. Solitary fibrous tumor of the pleura: a cytogenetic study. Cancer Genet Cytogenet 1999;111:169-71.

23. Miettinen M, el Rifai W, Sarlomo-Rikala M, et al. Tumor sizerelated DNA copy number changes occur in solitary fibrous tumors but not in hemangiopericytomas. Mod Pathol 1997;10:1194-200.

24. Thompson LD, Wieneke JA, Miettinen M, et al. Spindle cell (sarcomatoid) carcinomas of the larynx: a clinicopathologic study of 187 cases. Am J Surg Pathol 2002;26:153-70.

25. Loos BM, Wieneke JA, Thompson LD. Laryngeal angiosarcoma: a clinicopathologic study of five cases with a review of the literature. Laryngoscope 2001;111:1197-202.

26. Knott PD, Gannon FH, Thompson LDR. Mesenchymal chondrosarcoma of the sinonasal tract: A clinicopathologic study of 13 cases with a review of the literature. Laryngoscope 2003;113:783-90.

27. Thompson LD, Miettinen M, Wenig BM. Sinonasal-type hemangiopericytoma: a clinicopathologic and immunophenotypic analysis of 104 cases showing perivascular myoid differentiation. Am J Surg Pathol 2003;27:737-49.

28. Wenig BM. Tumours of the hypopharynx, larynx and trachea: mucosal malignant melanoma. In: Barnes EL, Eveson JW, Reichart P, Sidransky D, editors. Pathology and genetics of tumours of the head and neck, world health organization classification of tumours. Kleihues P, Sobin LH, series editors. Lyon, France: IARC Press, 2005:160-1.

29. Thompson LDR, Fanburg-Smith JC. Tumours of the hypopharynx, larynx and trachea: benign soft tissue tumours. In: Barnes EL, Eveson JW, Reichart P, Sidransky D, editors. Pathology and genetics of tumours of the head and neck, world health organization classification of tumours. Kleihues P, Sobin LH, series editors. Lyon, France: IARC Press, 2005:152-5.

30. Wenig BM. Tumours of the hypopharynx, larynx and trachea: inflammatory myofibroblastic tumour. In: Barnes EL, Eveson JW, Reichart P, Sidransky D, editors. Pathology and genetics of tumours of the head and neck, world health organization classification of tumours. Kleihues P, Sobin LH, series editors. Lyon, France: IARC Press, 2005:150-1. 